\title{
Effects of Foot Modelling on the Human Ankle Kinematics and Dynamics
}

\author{
Farnood Gholami ${ }^{a, 1}$, Rosa Pàmies-Vilà ${ }^{b, 2}$, József Kövecses $^{a, 3}$, Josep M. Font-Llagunes $^{b, 4}$ \\ ${ }^{a}$ Department of Mechanical Engineering and Centre for Intelligent Machines, McGill University \\ 817 Sherbrooke Street West, Montreal, Quebec, H3A 2K6, Canada \\ ${ }^{b}$ Department of Mechanical Engineering and Biomedical Engineering Research Centre, \\ Universitat Politècnica de Catalunya, Diagonal 647, 08028 Barcelona, Catalunya, Spain
}

\begin{abstract}
In this study, effects of some of the foot modelling assumptions on the ankle kinematics and dynamics are investigated based on the experimental data. For the kinematics analysis, the appropriateness of the stationary axis of rotation of the human ankle flexion is examined. Moreover, an interpolated function which is capable of predicting the directional changes of this axis is proposed. For the dynamics analysis, two main modelling assumptions of the number of the foot segments and the dimension of the foot model are the subject of the study. To this end, the ankle joint torque and power are selected as the comparison indicators and inverse dynamics analyses are carried out. The analyses show that the number of segments of the foot model does not have a considerable effect on the calculated ankle joint torque. On the other hand, the calculated ankle power is highly affected by both of the segmentation and the dimension of the foot model.
\end{abstract}

Keywords: Human Foot Modelling, Ankle Kinematics, Ankle Dynamics

\section{Introduction}

In the past years, different foot models for gait kinematics and dynamics studies have been introduced in the literature [6, 12, 18, 20, 23, 25, 27, 30, 31]. In most of these human gait analyses, ankle joint has been modelled based on a revolute joint [15, 19, 27], two revolute joints with non-orthogonal axes of rotations [7, 9, 24], or a spherical joint [26, 30, 31]. The popularity of modelling the ankle joint with the lower kinematics pairs are mainly due to their less-complex kinematics behaviour which make them simpler to be implemented in the human gait simulations and analyses. On the other hand, the applicability and appropriateness of these ankle models for the specific applications of interest are not well validated. More rigorous ankle kinematics analyses reported that the instantaneous axis of rotation (IAR) of the foot with respect to the tibia does not have a fixed orientation and location during the ankle rotation, even in the cases of pure flexion, pronation/supination or internal/external rotation performances [21, 22]. A mean orientation is usually selected when the ankle axis of rotation is modelled by a revolute joint.

Furthermore, on the foot modelling side, different models are proposed and used in the literature. Several studies have been done based on the point or circular shape foot models which cannot well capture the contact properties of the gait [12, 14, 28]. One-segment [5, 31], two-segment [27, 30], or three-segment foot models [6]

\footnotetext{
${ }^{1}$ E-mail: farnood.gholami@mail.mcgill.ca

${ }^{2}$ E-mail: rosa.pamies@upc.edu

${ }^{3}$ E-mail: jozsef.kovecses@mcgill.ca

${ }^{4}$ E-mail: josep.m.font@upc.edu
} 
are also proposed in the literature. Some studies suggested more segments for the foot [18, 20, 23, 25], for instance, the eight-segment foot by MacWilliams et al. [18]. Most of the multi-segment foot models have been used for kinematics analysis of the foot. Very few investigations have been done on the foot dynamics. Using multi-segment foot models to capture kinematics of the gait requires enough attention and study to appropriately place markers on the foot. As foot inter-segment movements are mostly in a narrow range, the marker placement should be well studied in these models to avoid drastic measurement errors. Furthermore, as the motion of some segments cannot be directly captured, approximations are used which the result is error-prone. Although the human gait can be simulated using the introduced models, still more improved models are needed as many simulations are for single step and not multiple step gait cycles [2, 15]. This drawback can be due to the fact that, in some situations, existing models may predict unrealistic foot kinematics and dynamics. Furthermore, although the three-dimensional multi-segment human model, as the most complex model, exists, a simple foot model consisting of one segment is mostly used for dynamics analysis [19, 31]. This might not fully characterize different phenomena in the human walking.

Foot models are mainly developed based on the following three assumptions: type of the ankle joint, number of segments, and dimension of the model. Conclusions have been drawn using these models while not enough justifications on the appropriateness of the utilized models were usually provided. Sensitivity of the predicted behaviours of these foot models with respect to each of the assumptions has not been completely understood. In the current paper, the aim is to further study the foot models which are widely used in the literature for certain kinematics and dynamics analyses. To this end, for the ankle joint kinematics, the leg-foot flexion motion is further analyzed in order to better understand the complex behaviour of the ankle during the gait. The ankle flexion motion is studied as the gait is accomplished mostly by this mode of rotation. An interpolated function to characterize the IAR of the foot with respect to the tibia is proposed. This function can be easily personalized and used for any other subject under the study. For dynamics studies, an inverse dynamics analysis is performed by calculating the ankle joint torque and power, as two comparison indicators. Comparison between the predicted results by these models are carried out. This can better demonstrate the differences in the predicted dynamics when different assumptions are made for foot modelling. Furthermore, by the aid of these analyses, more insight into drawbacks of these models and the appropriateness of them for different applications in the gait analysis can be gained.

\section{Kinematics and Dynamics Formulation}

Different methods to calculate the direction of the Instantaneous Axis of Rotation (IAR) which is in the same direction as the angular velocity of a rigid body exist in the literature. In this study, the angular velocity of a rigid body is calculated based on positions and velocities of three non-collinear points of the body, denoted by $\mathbf{p}_{1}, \mathbf{p}_{2}, \mathbf{p}_{3}$ and $\dot{\mathbf{p}_{1}}, \dot{\mathbf{p}_{2}}, \dot{\mathbf{p}_{3}}$, respectively. These positions and velocities are stored in matrices as $\mathbf{P}=\left[\begin{array}{lll}\mathbf{p}_{1}-\mathbf{c} & \mathbf{p}_{2}-\mathbf{c} & \mathbf{p}_{3}-\mathbf{c}\end{array}\right]$ and $\dot{\mathbf{P}}=\left[\begin{array}{lll}\dot{\mathbf{p}_{1}}-\dot{\mathbf{c}} & \dot{\mathbf{p}_{2}}-\dot{\mathbf{c}} & \dot{\mathbf{p}_{3}}-\dot{\mathbf{c}}\end{array}\right]$, where $\mathbf{c}=\frac{1}{3} \sum_{i=1}^{3} \mathbf{p}_{i}$ and $\dot{\mathbf{c}}=\frac{1}{3} \sum_{i=1}^{3} \dot{\mathbf{p}_{i}}$. Now, if we define $\mathbf{R}=\mathbf{P} \mathbf{P}^{T}$, and $\mathbf{J}=\operatorname{tr}(\mathbf{R}) \mathbf{E}-\mathbf{R}$, where $\mathbf{E}$ is the identity matrix, $t r$ and vect are the trace and vector operators respectively, the angular velocity of the body can be expressed as $[3]$

$$
\boldsymbol{\omega}=2 \mathbf{J}^{-1} \operatorname{vect}\left(\dot{\mathbf{P}} \mathbf{P}^{T}\right) .
$$

The ankle joint torque and power are common indicators in the human gait dynamics analysis [8, 11]. For a one-segment foot model, based on the application of the angular momentum theorem on the foot segment, the ankle joint torque can be formulated as

$$
\mathbf{T}_{a}+\mathbf{T}_{r}+\mathbf{r}_{c a} \times \mathbf{F}_{a}=\mathbf{I} \dot{\boldsymbol{\omega}}+\boldsymbol{\omega} \times(\mathbf{I} \boldsymbol{\omega})
$$

where $\mathbf{T}_{a}$ is the ankle torque, $\mathbf{T}_{r}$ is the resultant ground reaction moment at the center of mass of the segment, $\mathbf{r}_{c a}$ is the position vector from the center of mass to the ankle position, $\mathbf{F}_{a}$ is the ankle joint force, $\mathbf{I}$ is the foot tensor of inertia at the center of mass and $\boldsymbol{\omega}$ is the angular velocity vector of the foot segment. If 
the foot-ground contact interaction is measured through force plates, as is the usual case in inverse dynamics studies, the resultant ground reaction moment can be expressed as

$$
\mathbf{T}_{r}=\mathbf{T}_{f}+\mathbf{r}_{c f} \times \mathbf{F}_{f}
$$

where $\mathbf{T}_{f}$ is the ground reaction moment with respect to the center of the force plate, $\mathbf{r}_{c f}$ is the position vector from the center of mass to the center of the force plate and $\mathbf{F}_{f}$ is the foot-ground contact force. The formulation can be extended for the two-segment foot as

$$
\begin{aligned}
& \mathbf{T}_{a}+\mathbf{T}_{r}+\mathbf{r}_{c_{1} a} \times \mathbf{F}_{a}+\mathbf{r}_{c_{1} m} \times \mathbf{F}_{m}-\mathbf{r}_{c_{2} m} \times \mathbf{F}_{m}= \\
& \mathbf{I}_{1} \dot{\boldsymbol{\omega}}_{1}+\boldsymbol{\omega}_{1} \times\left(\mathbf{I}_{1} \boldsymbol{\omega}_{1}\right)+\mathbf{I}_{2} \dot{\boldsymbol{\omega}}_{2}+\boldsymbol{\omega}_{2} \times\left(\mathbf{I}_{2} \boldsymbol{\omega}_{2}\right)
\end{aligned}
$$

where $\mathbf{r}_{c_{1} a}$ is the position vector from the center of mass of the hindfoot (segment 1) to the ankle position, $\mathbf{r}_{c_{1} m}$ is the position vector from the center of mass of the first foot segment to the metatarsal position, $\mathbf{F}_{m}$ is the metatarsal reaction force, $\mathbf{T}_{m}$ is the metatarsal torque, $\mathbf{r}_{c_{2} m}$ is the position vector from the center of mass of the forefoot (segment 2) to the metatarsal position, $\boldsymbol{\omega}_{\boldsymbol{i}}$ is the vector of angular velocity of the $i$ th foot segment, $\mathbf{I}_{i}$ is the moment of inertia of the $i$ th foot segment, $\mathbf{F}_{s_{2}}, \mathbf{a}_{2}$, and $\mathbf{g}$ are the weight, acceleration of the second segment and the gravity vector respectively. Due to low inertial effects of the forefoot segment, the ground reaction forces are assumed to act on the hindfoot only. The ankle joint torque for foot models with more segments can be similarly derived as well. Power done by muscles acting on the ankle joint can be formulated as, $P=\mathbf{T}_{a} \cdot \boldsymbol{\omega}_{\text {rel }}$, where $\boldsymbol{\omega}_{\text {rel }}$ is the relative angular velocity of the foot with respect to the tibia.

\section{Motion Capture and Data Analysis}

To capture the leg and foot motion, which is of main interest to us, the skin markers need to be placed on them based on the chosen foot model as well as the marker placement protocol. This means that even for the same foot model, different kinematics can be obtained due to the differences in the chosen anatomical landmarks where markers are placed. Marker protocols are selected based on the model and the anatomy of the foot and the leg. While number of segments of the model determines the minimum required number of markers, not a unique marker placement protocol would exist for the same model. The landmarks for the tibia are usually identical in all different protocols which are selected as tibial tuberosity, lateral tibia, and medial tibia (Fig 1). The main difference stemmed from the marker attachment on the foot. For one-segment foot models, usually three markers are attached to the medial calcaneus, lateral calcaneus, and between the bases of the second and third metatarsal joints [29] (p1: Fig 1(a)). For two-segment foot models, two protocols are more popular. For the hindfoot segment, while the first two markers in both protocols are mounted on the medial and lateral calcaneus, the third marker could be attached either on the posterior calcaneus (p2: Fig 1(b)) or the base of the fifth metatarsal joint (p3: Fig 1(c)) [26, 29]. The former protocol can be used for the hindfoot of three-segment foot models as well [6]. As the ankle dynamics is under the study, well capturing the hindfoot motion is more critical than the forefoot. For the forefoot, the landmarks to place the markers are selected as medial calcaneus, anterior tubercle calcaneus, and between and $10 \mathrm{~mm}$ proximal to 2nd and 3rd metatarsal. A combination of all mentioned marker protocols are considered for our motion capture trial. As a result, the data associated with different subsets of these markers can be selected and used for analyses of each of the foot models. In this way, the utilized data in the analyses of different foot models are captured at the same trial which make the comparison more meaningful.

For our analyses, the motion was recorded by means of 12 OptiTrack FLEX:V100R2 cameras sampling at $100 \mathrm{~Hz}$. Two force plates AMTI AccuGait located on a walkway were used to measure the ground reaction forces. The force plate data are synchronized with the stored trajectories. Both set of data are expressed in a same orthogonal global frame. Markers were attached to the human body according to the defined model segments and the selected protocol. Two sets of experiments are conducted. First, a seated $71 \mathrm{~kg}$ healthy male adult with no history of gait abnormality is asked to perform a flexion tibia-foot motion for 5 complete 
cycles. The fifth cycle is excluded and considered as the control data. A tibia reference frame centred at marker 8 is defined based on the following unit vectors

$$
\mathbf{e}_{y}=\frac{\mathbf{r}_{86} \times \mathbf{r}_{84}}{\left\|\mathbf{r}_{86} \times \mathbf{r}_{84}\right\|}, \quad \mathbf{e}_{x}=\frac{\mathbf{r}_{84}}{\left\|\mathbf{r}_{84}\right\|}, \quad \mathbf{e}_{z}=\mathbf{e}_{x} \times \mathbf{e}_{y}
$$

in which $\mathbf{r}_{84}=\mathbf{r}_{4}-\mathbf{r}_{8}$ and $\mathbf{r}_{86}=\mathbf{r}_{6}-\mathbf{r}_{8}$, where $\mathbf{r}_{i}$ is the position vector associated with the marker $i$. Also, an ankle reference frame is defined as follow

$$
\mathbf{u}_{x}=\mathbf{r}_{73}, \quad \mathbf{u}_{z}=\frac{\mathbf{r}_{23} \times \mathbf{u}_{x}}{\left\|\mathbf{r}_{23} \times \mathbf{u}_{x}\right\|}, \quad \mathbf{u}_{y}=\mathbf{u}_{z} \times \mathbf{u}_{x}
$$

Second, the subject is asked to walk normally through the walkway and the gait is captured via the system. This is used for the ankle dynamics analyses.

During gait, skin motion and muscle deformations cause relative motions of the markers with respect to the skeleton. This is regarded as one of the most critical sources of error in the human movement analysis. Different methods can be used to reduce the noise in the data, e.g., digital filters, splines, or singular spectrum analysis techniques [1]. The position signal contains a noise that has a similar frequency response as the motion itself. In this work, the captured data is filtered via a sixth order Butterworth method, with the cut-off frequency of $10 \mathrm{~Hz}$, to smooth the trajectories from the noises. On the other hand, kinematic errors (that are intrinsic to the motion capture process) affect the estimation of the joints position, and lead to violations of the kinematic constraints. The existence of such kinematic errors are mainly due to the measurement errors of the motion capture system, skin artifact effects, and inter-bone movements which are not modelled. In order to avoid such a kinematic inconsistency problem, a new set of coordinates is calculated by imposing the kinematic consistency at position level through a minimization problem. To this end, each set of markers which are assumed to be attached to the same body segment, needs to obey the general rigid body assumption. This means that markers on the same rigid body should preserve the relative distances during the gait. By enforcing the markers to satisfy this set of constraints, new marker trajectories $(\tilde{\mathbf{q}})$ can be found in which the skin artifact effects are reduced and the kinematic consistency is ensured. The kinematic consistency at position level can be achieved through a minimization problem as [1]

$$
\min _{\mathbf{q}} V=\frac{1}{2}\left(\tilde{\mathbf{q}}-\mathbf{q}_{f}\right)^{T} \mathbf{W}\left(\tilde{\mathbf{q}}-\mathbf{q}_{f}\right) \quad \text { s.t. } \quad \boldsymbol{\Phi}(\tilde{\mathbf{q}})=\mathbf{0}
$$

where $\boldsymbol{\Phi}$ is the constraint equations vector, $\mathbf{q}_{f}$ is the filtered position of the markers in the inertial reference frame, $\tilde{\mathbf{q}}$ is the new marker positions assuring kinematic consistency, and $\mathbf{W}$ is a weighting diagonal matrix. Finally, a fitted spline function on the marker trajectories is differentiated to obtain the velocities and accelerations of the points.

To carry out dynamics calculations, all the required inertial properties and segment dimensions are selected based on the existing anthropometric data [10] with some adjustments (Table 10). The moment of inertias are expressed in the local axes. Furthermore, the location of the ankle joint and the center of mass of the foot segments are both estimated based on the formulations introduced in [29].

\section{Results and Discussions}

\subsection{Ankle Kinematics}

Variations of the ankle IAR during the foot flexion expressed in the tibia reference frame are illustrated in Fig. 2. The non-stationary nature of the ankle IAR indicates that considering a revolute joint for the tibia-foot flexion motion is not the most appropriate model and can give rise to considerable errors. Here, we find an interpolated function which can characterize the direction of the IAR at each flexion angle (Figures 3a. 3c). By this approach, instead of using a stationary axis of rotation (based on the mean directions over the range of flexion), a non-stationary axis can be considered in which a function predicts its direction at 


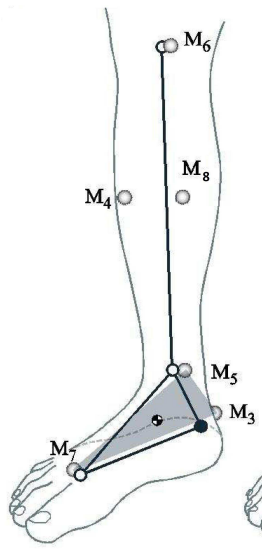

(a)

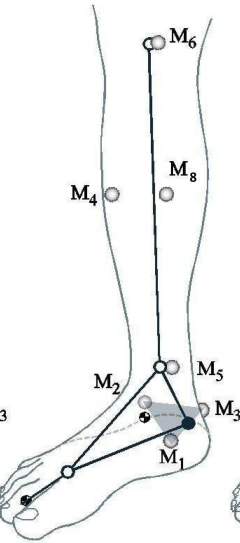

(b)

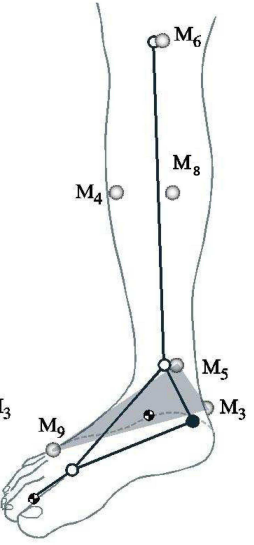

(c)

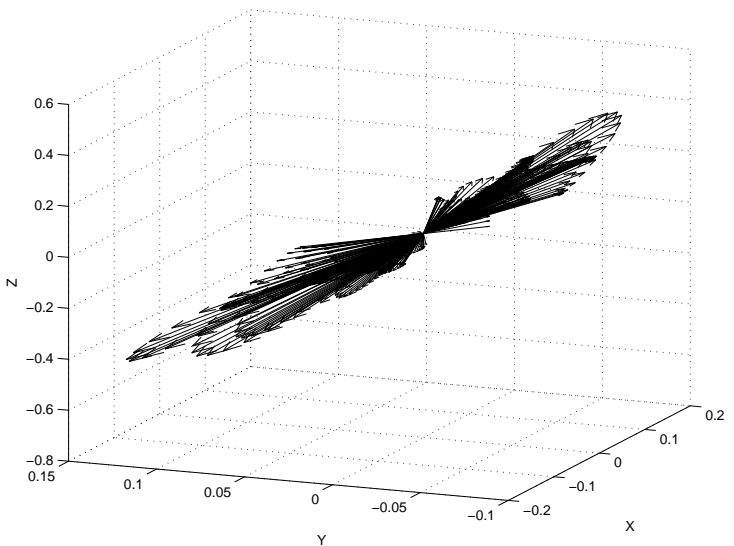

Figure 2: Relative angular velocity of the foot with Figure 1: p1, p2, and p3 marker placement protocols respect to the tibia expressed in the tibia reference for ankle dynamic analysis

frame captured for four cycles

Table 1: Foot Anthropometric parameters

\begin{tabular}{ccc}
\hline \hline Anthropometric measurement & Value & Unit \\
\hline Hindfoot mass & 0.7750 & $\mathrm{~kg}$ \\
Forefoot mass & 0.1938 & $\mathrm{~kg}$ \\
Foot length & 0.260 & $\mathrm{~m}$ \\
Malleolus height & 0.06 & $\mathrm{~m}$ \\
Malleolus width & 0.074 & $\mathrm{~m}$ \\
Hindfoot moment of inertia in ankle frame & $0.0011,0.0033,0.0033$ & $\mathrm{~kg} \cdot \mathrm{m}^{2}$ \\
Forefoot moment of inertia in ankle frame & $0.0008,0.0030,0.0034$ & $\mathrm{~kg} \cdot \mathrm{m}^{2}$
\end{tabular}

each flexion angle. This can more realistically characterize the ankle kinematics. The selected interpolated function for each $\mathrm{x}, \mathrm{y}$, and $\mathrm{z}$ components of the ankle IAR is based on the sum of sin functions as

$$
f(\theta)_{n}=a_{1_{n}} \sin \left(b_{1_{n}} \theta+c_{1_{n}}\right)+a_{2_{n}} \sin \left(b_{2_{n}} \theta+c_{2_{n}}\right)+a_{3_{n}} \sin \left(b_{3_{n}} \theta+c_{3_{n}}\right), \quad n=x, y, z
$$

where $f(\theta)_{n}(n=x, y, z)$ is the function which characterizes the ankle flexion IAR in each of the $x, y, z$ directions expressed in the tibia reference frame, $\theta$ is the angle between the foot and the tibia defined as the angle between $\mathbf{r}_{56}$ and $\mathbf{r}_{57}$. Furthermore, $a_{i_{n}}, b_{i_{n}}, c_{i_{n}},(i=1, \ldots, 3)$ are coefficients obtained based on the experimental data. For any other subject, with different anatomical specifications, these coefficients need to be experimentally determined. For the subject under the study, these coefficients have been found using MATLAB ${ }^{\circledR}$ curve fitting toolbox capabilities(Table 2). Furthermore, the mean direction of the ankle axis of rotation is determined. This direction is usually used for the revolute joint models at the ankle. Finally, the ankle IARs based on the set of the control data are determined as well. The IAR suggested by the interpolated formulation, as well as the IAR of the control data set, and the mean direction of the ankle axis are illustrated in Figures 4 a $4 \mathrm{C}$. The interpolated function shows acceptable agreement with the new set of experimental control data, while the mean axis cannot capture the natural behaviour of the foot-tibia motion. Furthermore, the Residual Sum of Squares (RSS) for both the interpolated function and the mean direction are calculated with respect to the control data (Table 3). The RSS indicates how much the interpolated function or the mean value is deviated from the set of experimental control data. Using the proposed function, the RSSs associated with each direction are much lower than the mean directions. This further emphasizes that the proposed approach can characterize the ankle flexion kinematics more 
realistically. This approach can be easily personalized for any other subject and be used in different studies and applications.

Table 2: Coefficients of the interpolated function

\begin{tabular}{cccccccccc}
\hline \hline & $a_{1_{n}}$ & $a_{2_{n}}$ & $a_{3_{n}}$ & $b_{1_{n}}$ & $b_{2_{n}}$ & $b_{3_{n}}$ & $c_{1_{n}}$ & $c_{2_{n}}$ & $c_{3_{n}}$ \\
\hline$n=x$ & 1.144 & 0.1164 & -4.421 & 0.8767 & 0.1356 & 3.098 & 0.03752 & 0.4934 & 15.31 \\
$n=y$ & 0.2478 & 0.04843 & 5.724 & 0.01363 & 0.2309 & -5.225 & 0.02239 & 0.4632 & 1.709 \\
$n=z$ & 1.062 & 0.002489 & 7.117 & 0.05481 & 0.1208 & 3.525 & 0.01832 & 0.3801 & -7.242
\end{tabular}

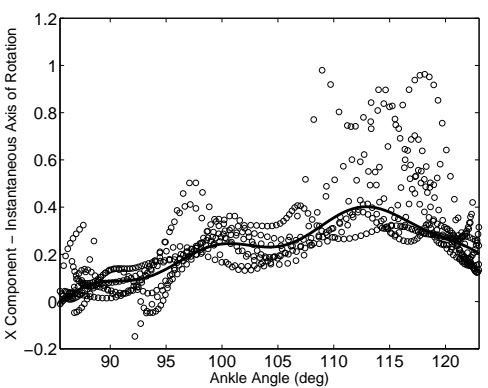

(a) X component of the ankle IAR

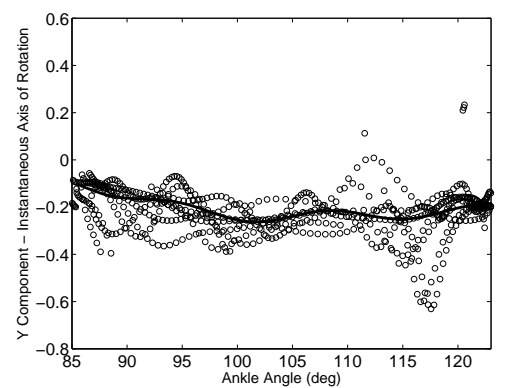

(b) Y component of the ankle IAR

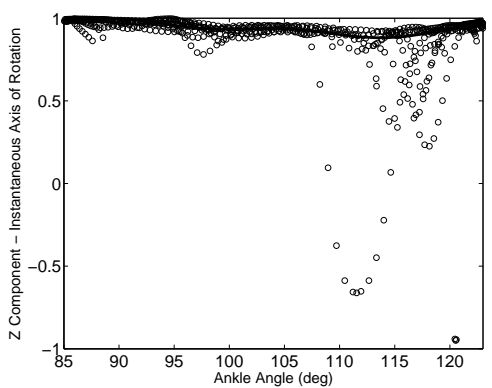

(c) Z component of the ankle IAR

Figure 3: IAR interpolation direction for the ankle flexion motion - hollow circles are associated with the experimental data and solid lines are the interpolated curves

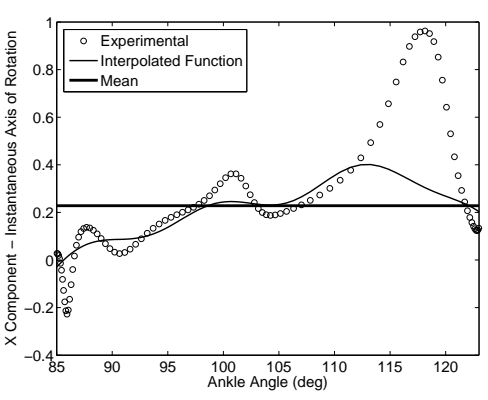

(a) X component

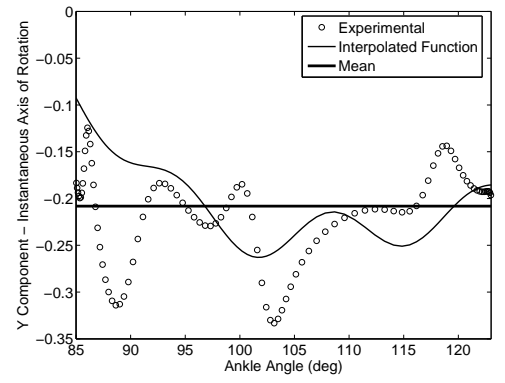

(b) Y component

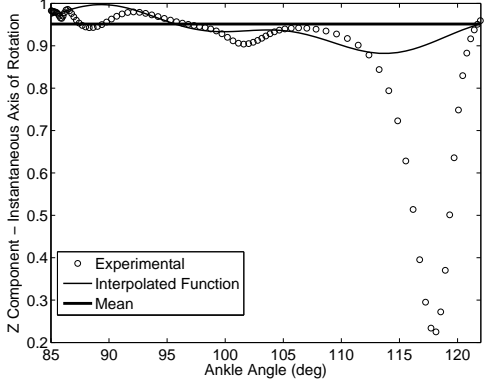

(c) Z component

Figure 4: IAR Interpolation, mean, and experimental directions

Table 3: Residual Sum of Squares

\begin{tabular}{ccc}
\hline \hline & Interpolated Function & Mean Direction \\
\hline X component & 1.3649 & 2.2873 \\
Y component & 0.0516 & 0.1018 \\
Z component & 0.6391 & 0.8573
\end{tabular}




\subsection{Ankle Dynamics and Energetics}

Here, the effects of the main foot modelling assumptions on the ankle dynamics are investigated. These assumptions are the foot segmentation and the dimension of the model. To analyze the effects of the foot segmentation, two models, namely, one-segment and two-segment foot models are selected. For the twosegment foot model, two marker placement protocols on the hindfoot are mostly used in the literature ( 22 $\& \mathrm{p} 3)$.
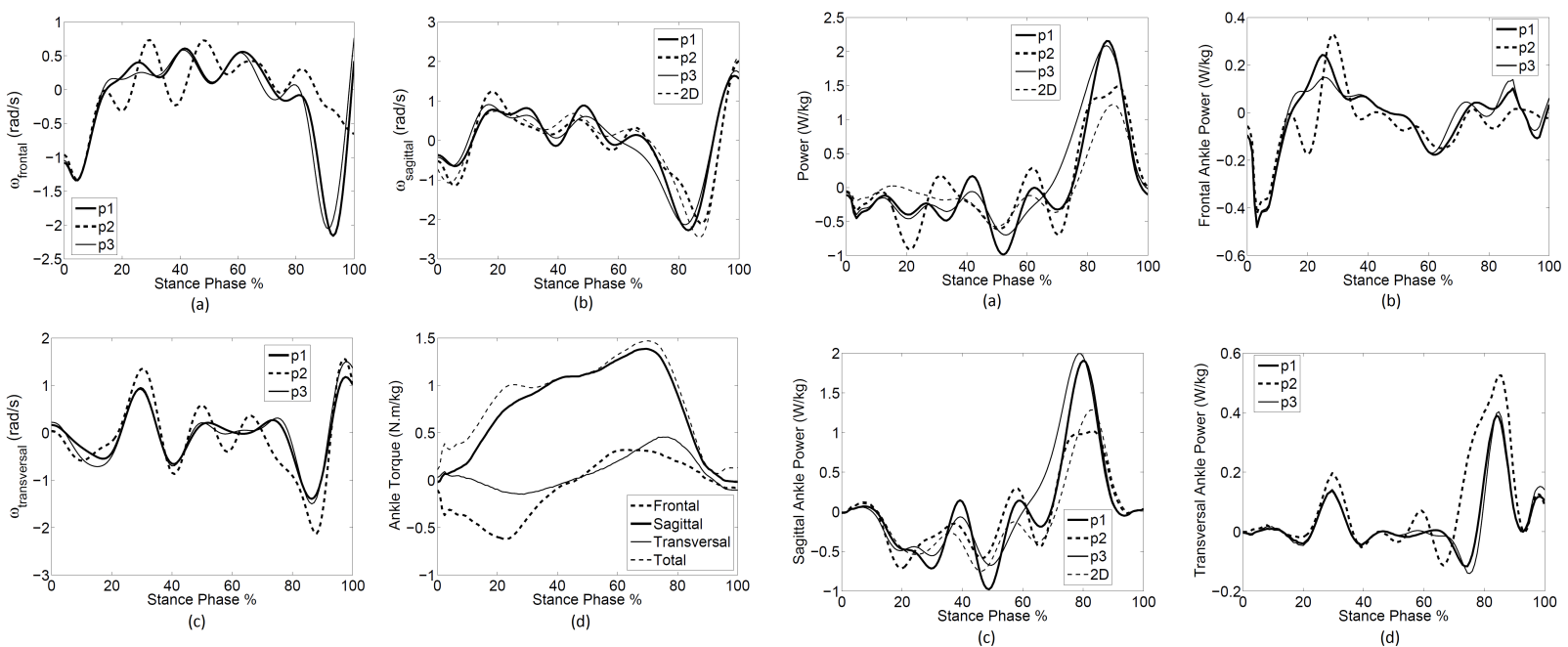

Figure 5: (a)-(c): Frontal, sagittal, and transversal com- Figure 6: (a): Ankle powers for protocols p1-p3, and twoponents of the relative angular velocity of foot with respect dimensional models, (b)-(d): pronation/supination, flexto tibia, (d): frontal, sagittal, transversal components, and ion, and adduction/abduction ankle powers for protocols norm of ankle torque for three-dimensional foot model p1-p3

The ankle joint torque and power are calculated for the gait stance phase. Considering one- and twosegment foot models, almost no considerable differences in the ankle torques are observed while the calculated ankle powers are considerably different (Fig. 6(a)). Although considering multiple segments in the foot models can better capture the foot inter-segment movements and moments [4, 11, 17, 18, 27, 29], our study shows that this modelling assumption does not have considerable effects on the ankle joint torque calculations in an inverse dynamics approach. Based on the dynamic formulations in Eqs. (2) and (4), and considering the fact that the foot segments contain light masses (Table 1), the foot segmentation should not have much effect on the calculated ankle joint torque. Our experimental results show that while the order of magnitude of the inertial effects are $10^{-1}$ N.m, the moment of the ground contact forces could be as big as 100 N.m. As a result, for those type of analyses with specific interest on the ankle torques, foot segmentation does not influence the results. In [8], this was shown by experimental results based on the motion capture data and not a solid dynamic analysis for the justification. Our study suggests that multi-segment foot models do not bring much accuracy to the ankle dynamics but to the inter-segment kinematics and joint dynamics.

It should be noted that analyzing two-segment foot models can give insight into the behaviour of models with more segments too. Increasing the number of segments does not affect the ankle torque due to the mentioned negligible inertial effects of the additional forefoot segments. On the other hand, in both of the two- and multi-segment foot models, usually the calcaneus bone is selected as the hindfoot segment. As a result, the additional segments introduced in these models have the main contribution on inter-segment effects. Having this said, as the ankle power depends on the angular velocity of the hindfoot segment and not the other forefoot segments, the calculated ankle powers for the two- or multi-segment foot models should not differ considerably.

Next, the effects of the dimension of the model on the predicted ankle torque and power are investigated. 
To this end, two-dimensional and three-dimensional foot models are considered. For the three-dimensional foot model, the contribution of each direction to the total power ankle are illustrated individually as well (Fig. 6(b)-(d)). These are calculated based on the projected angular velocities and ankle torques into the corresponding planes. In both models, the foot is considered as one segment which is connected to the tibia via a revolute or spherical joint, respectively. For the two-dimensional model, less number of markers (three markers located on the tibia and the foot, namely, markers 5, 6, and 7) are needed (Fig. 1). In this model, the angular velocity is calculated based on the rate of change of the ankle angle. As it can be observed (Fig. 5(d) and Fig. 6(a)) the dimension of the model affects both the calculated ankle torque and power. While the two-dimensional foot model is able to only capture the sagittal ankle torque and power, the other two torque and power components, namely the frontal and the transversal, can only be captured via using the three-dimensional foot models. The existence of these non-negligible values of the frontal and transversal ankle torque and power, which contribute to the total values, could further highlight the effects of this modelling assumption on the predicted foot dynamics. If the norm of the total ankle torque and the total power for the three-dimensional model is compared with the two-dimensional one, the differences at some instances can be as big as $30 \%$ and $50 \%$ respectively. Two-dimensional foot models are commonly used in the literature due to the simplifications they bring for the kinematic and dynamic formulations, as well as the motion capture procedures 13, 31]. This study shows that both the calculated ankle torques and powers could be very much affected by the dimension of the model. This is due to the off-sagittal plane components of both the foot movements and the ground reaction forces and moments.

Finally, this study further emphasizes that the calculated power is not only sensitive to the foot model, but also to the marker placement protocol. Consequently, for the same foot model, different angular velocities, and as a result powers, can be obtained when different marker protocols are used. Considering two sets of foot marker protocols labelled as p2 and p3, both for the same two-segment foot model, the calculated foot angular velocity, and consequently ankle power, can be very much different (Fig. 5(a)-(c) and Fig. 6(a)). This further highlights the importance of selecting an appropriate marker placement protocol for the ankle kinematics and dynamics analyses.

\section{Conclusions}

Effects of some of the foot modelling assumptions on the ankle kinematics and dynamics were investigated in this study. The analyses were carried out based on the experimental data of a motion capture system. The appropriateness of modelling the human ankle joint based on a stationary axis of rotation was investigated. Moreover, we proposed an interpolated function which is capable of predicting the directional changes of the ankle axis during the foot flexion. Furthermore, two main modelling assumptions of the number of the foot segments and the dimension of the foot model were the subject of the foot dynamics analyses. The ankle joint torque and power were selected as the comparison indicators and inverse dynamics analyses were carried out. We showed that the number of segments of the foot model does not have a considerable effect on the calculated ankle joint torque while the ankle power can be considerably affected by both of the foot segmentation and the dimension of the foot model.

\section{References}

[1] F. J. Alonso, J. Cuadrado, U. Lugris, P. Pintado. A compact smoothing-differentiation and projection approach for the kinematic data consistency of biomechanical systems. Multibody System Dynamics, 24(1), 67-80, 2010.

[2] F. C. Anderson, and M. G. Pandy. Dynamic Optimization of Human Walking. ASME J. Biomech. Eng., 123(5), 381-390, 2001.

[3] J. Angeles. Fundamentals of Robotic Mechanical Systems: Theory, Methods, and Algorithms. Springer-Verlag, 2003.

4. D. A. Brueninga, K. M. Cooney, F. L. Buczek. Analysis of a kinetic multi-segment foot model part II: Kinetics and clinical implications. Gait Posture. 35, 535-540, 2012.

[5] F. L. Buczek, M. R. Walker, M. J. Rainbow, K. M. Cooney, J. O. Sanders. Impact of mediolateral segmentation on a multi-segment foot model. Gait \& Posture, 23, 519-522, 2006.

[6] M.C. Carson, M.E. Harringtonb, N. Thompsonb, J.J. Ó Connora, T.N. Theologisc. Kinematic analysis of a multi-segment foot model for research and clinical applications: a repeatability analysis. Journal of Biomechanics, 34, $1299-1307,2001$.

[7] M. Dettwyler, A. Stacoff, I. A. Kramers-de Quervain, E. Stüssi. Modelling of the ankle joint complex. Reflections with regards to ankle prostheses, Journal of Foot and Ankle Surgery, 10(3),109-119, 2004. 
[8] P. C. Dixon, H. Böhm, L. Dö. Derlein ankle and midfoot kinetics during normal gait:a multi-segment approach, Journal of Biomechanics, 45, 1011-1016, 2012.

[9] J. Dul, G.E. Johnson. A kinematic model of the human ankle, Journal of Biomedical Engineering, 7(2),137-143, 1985.

[10] R. Dumas, L. Chéze, J.-P. Verriest Adjustments to McConville et al. and Young et al. body segment inertial parameters, Journal of Biomechanics,40(3), 543-553, 2007.

[11] J. J. Eng, D. A. Winter. Kinetic analysis of the lower limbs during walking: what information can be gained from a 3-dimensional model? Journal of Biomechanics, 28(6), 753-758, 1995.

[12] J.M. Font, J. Kövecses. Dynamics of heel strike in bipedal systems with circular feet. Proceedings of The Second European Conference on Mechanism Science EUCOMES, 455-462, Cassino, Italy, 2008.

[13] C. Frigo, P. Crenna, L. M. Jensen. Moment-Angle Relationship at Lower Limb Joints during Human Walking at Different Velocities. Journal of Electromyogr Kinesiol. , 6(3), 177-90, 1996

[14] M. Garcia, A. Chatterjee, A. Ruina, M. Coleman. The simplest walking model: stability, complexity, and scaling. Journal of Biomechanical Engineering, 120(2), 281-288, 1998.

[15] K. G. M. Gerritsen, A. V. Bogert, B. M. Nigg. Direct dynamics simulation of the impact phase in heel-toe running. Journal of Biomechanics, 28, 661-668, 1995.

[16] A. H. Hansen, D. S. Childress, S. C. Miff, S. A. Gard, K. P. Mesplay. The human ankle during walking: implications for design of biomimetic ankle prostheses. Journal of Biomechanics, 37, 1467-1474, 2004.

[17] S. Hwang, Y.H Kim. Foot motion on hallux valgus patients: a multi-segment foot model. World Congress on Medical Physics and Biomedical Engineering, 14(17), 2969-2972, 2007.

[18] B. A. MacWilliams, M. Cowley, D. E. Nicholson. Foot kinematics and kinetics during adolescent gait. Gait \& Posture, 17, 214-224, 2003.

[19] M. Millard, J. McPhee, E. Kubica. Multi-step forward dynamic gait simulation. In: Bottasso CL, editor. Multibody Dynamics: Computational Methods and Applications, Springer, 25-43, 2009.

[20] M. Morlock and B. M. Nigg. Theoritical Considerations and Practical Results on the Influence of the Representation of the Foot for the Estimation of Internal Forces with Models. Clin. Biomechanics, 6, 3-13, 1991.

[21] A. Leardini, R. Stagni, J. J. O'Connor, Mobility of the subtalar joint in the intact ankle complex. Journal of Biomechanics, 34, 805-809, 2001.

[22] A. Lundberg, O. K. Svensson, The axes of rotation of the talocalcaneal and talonavicular joints. The Foot, 3(2),65-70, 1993.

[23] P. Lundgren, C. Nester, A. Liu, A. Arndt, R. Jones, A. Stacoff, P. Wolf, A. Lundberg. Invasive in vivo measurement of rear-, mid- and forefoot motion during walking, Gait \& Posture, 28, 93-100, 2008.

[24] S. H. Scott, D. A. Winter. Talocrural and talocalcaneal joint kinematics and kinetics during the stance phase of walking, Journal of Biomechanics, 24(8), 743-752, 1991.

[25] H. S. Stephen and D. A. Winter. biomechanical model of the human foot: kinematics and kinetics during the stance phase of walking. Journal of Biomechanics, 29 (9), 1091-1104, 1993.

[26] P. Smith, J. Humm, S. Hassani, G. Harris . Three Dimensional Motion Analysis of the Pediatric Foot and Ankle. Human Motion Analysis, G. F. Harris and P. A. Smith, Eds. Piscataway, NJ: IEEE Press, 351-369, 2000.

[27] D. J. Stefanyshyn, B. M. Nigg. Mechanical energy contribution of the metatarsophalangeal joint to running and sprinting Journal of Biomechanics, 3(11), 1081-1085, 1997.

[28. R.Q. van der Linde. Passive bipedal walking with phasic muscle contraction. Biological Cybernetics, 81, 227-237, 1999.

29. C. L. Vaughan, B. L. Davis, J. C. O'Connor. Dynamics of Human Gait. Human Kinetics Publishers, 1992.

30] R. Wang, C.K. Thur, E. M. Gutierrez-Farewik, P. Wretenberg, E. Broströmc. One year follow-up after operative ankle fractures: A prospective gait analysis study with a multi-segment foot model . Gait \& Posture, 31, 234-240 , 2010.

[31] M. Wojtyra. Multibody simulation model of human walking. Mechanics Based Design of Structures and Machines, 31(3), $357-379,2003$. 